PROCEEDINGS OF THE

AMERICAN MATHEMATICAL SOCIETY

Volume 134, Number 1, Pages 235-242

S 0002-9939(05)07958-X

Article electronically published on June 13, 2005

\title{
DISCRETENESS AND SIMPLICITY OF THE SPECTRUM OF A QUASILINEAR STURM-LIOUVILLE-TYPE PROBLEM ON AN INFINITE INTERVAL
}

\author{
PAVEL DRÁBEK AND ALOIS KUFNER \\ (Communicated by Carmen C. Chicone)
}

\begin{abstract}
We present sufficient conditions on the coefficients to get the discreteness and simplicity of the spectrum of a quasilinear Sturm-Liouville-type problem on an infinite interval. This condition appears to be necessary and sufficient for the compact embedding of certain weighted spaces. Our result generalizes those which are known from linear theory.
\end{abstract}

\section{INTRODUCTION}

Let $p>1$ be a real number and let $\varphi: \mathbb{R} \rightarrow \mathbb{R}$ be defined as $\varphi(s)=|s|^{p-2} s$ for $s \neq 0, \varphi(0)=0$. Let $r=r(t), c=c(t)$ be continuous and positive functions on $[0, \infty)$. For $x=x(t)$ defined on $[0, \infty)$ denote $x(\infty):=\lim _{t \rightarrow \infty} x(t)$. In this paper we will study the eigenvalue problem

$$
\left\{\begin{array}{l}
\left(r(t) \varphi\left(x^{\prime}(t)\right)\right)^{\prime}+\lambda c(t) \varphi(x(t))=0, \quad t \geq 0 \\
x^{\prime}(0)=0, x(\infty)=0
\end{array}\right.
$$

where $\lambda \in \mathbb{R}$ is a spectral parameter. By a solution of (1.1) we mean an absolutely continuous function $x=x(t)$, defined on $[0, \infty)$, such that $r \varphi\left(x^{\prime}\right) \in C^{1}[0, \infty)$, the equation in (1.1) is satisfied at every point and $x^{\prime}(0)=x(\infty)=0$. The parameter $\lambda$ is called an eigenvalue of (1.1) if this problem has a nontrivial (i.e. nonzero) solution (called an eigenfunction of (1.1)).

Our main result depends on the following condition on the weight functions (coefficients in the equation) $r$ and $c$ :

$$
\lim _{t \rightarrow \infty}\left(\int_{0}^{t} c(\tau) \mathrm{d} \tau\right)^{1 / p}\left(\int_{t}^{\infty} r^{1-p^{\prime}}(\tau) \mathrm{d} \tau\right)^{1 / p^{\prime}}=0
$$

Theorem 1.1. Let us suppose (1.2). Then the set of all eigenvalues of (1.1) forms an increasing sequence $\left\{\lambda_{n}\right\}_{n=1}^{\infty}$ such that $\lambda_{1}>0$ and

$$
\lim _{n \rightarrow \infty} \lambda_{n}=\infty \text {. }
$$

Received by the editors February 25, 2004 and, in revised form, August 30, 2004.

2000 Mathematics Subject Classification. Primary 34L05, 47E05, 34B40.

Key words and phrases. Discreteness of the spectrum, compact embeddings of the weighted spaces, minimax principles.

This research was supported by the Grant Agency of the Czech Republic, grant No. 201/03/0671, and by the Grant Agency of the Academy of Sciences of the Czech Republic, grant No. A1019305.

(C)2005 American Mathematical Society Reverts to public domain 28 years from publication 
Every eigenvalue $\lambda_{n}, n=1,2, \ldots$, is simple in the sense that there exists a unique normalized eigenfunction $x_{\lambda_{n}}$ associated with $\lambda_{n}$. Moreover, the eigenfunction $x_{\lambda_{n}}$ has precisely $n-1$ zeros in $(0, \infty)$. In particular, $x_{\lambda_{1}}$ does not change sign in $(0, \infty)$. For $n \geq 3$, between two consecutive zeros of $x_{\lambda_{n-1}}$ in $(0, \infty)$ there is exactly one zero of $x_{\lambda_{n}}$.

We shall also prove that every $\lambda_{n}, n=1,2, \ldots$, allows a variational characterization similar to the well-known Courant-Weinstein principle. For more details see Section 4.

Condition (1.2) is thus sufficient for extending some fundamental properties of Sturm-Liouville systems to weighted quasilinear problems on an infinite interval.

The following simple examples show that without (1.2) the assertion of Theorem 1.1 need not be true.

Example 1.2. Let $p=2, r \equiv c \equiv 1$. Then evidently there is no solution of

$$
x^{\prime \prime}(t)+\lambda x(t)=0, \quad x^{\prime}(0)=0, x(\infty)=0
$$

for any $\lambda \in R$. Note that in this case we have

$$
\left(\int_{0}^{t} c(\tau) \mathrm{d} \tau\right)^{1 / p}\left(\int_{t}^{\infty} r^{1-p^{\prime}}(\tau) \mathrm{d} \tau\right)^{1 / p^{\prime}} \equiv \infty, \quad t \in(0, \infty) .
$$

Example 1.3. Let $p=2, r(t)=(t+1)^{2}, c(t) \equiv 1$. Then we have

$$
\begin{aligned}
& \left(\int_{0}^{t} c(\tau) \mathrm{d} \tau\right)^{1 / p}\left(\int_{t}^{\infty} r^{1-p^{\prime}}(\tau) \mathrm{d} \tau\right)^{1 / p^{\prime}} \\
& \quad=\left(\int_{0}^{t} \mathrm{~d} \tau\right)^{1 / 2}\left(\int_{t}^{\infty} \frac{\mathrm{d} \tau}{(1+\tau)^{2}}\right)^{1 / 2}=\left(\frac{t}{1+t}\right)^{1 / 2} \rightarrow 1
\end{aligned}
$$

for $t \rightarrow \infty$. The initial value problem

$$
\left((t+1)^{2} x^{\prime}(t)\right)^{\prime}+\lambda x(t)=0, \quad x(0)=1, x^{\prime}(0)=0
$$

has the solution

for $\lambda=\frac{1}{4}$,

$$
x(t)=(t+1)^{-1 / 2}\left(1+\frac{1}{2} \log (t+1)\right)
$$

$x(t)=(t+1)^{-1 / 2}\left[\left(\frac{1}{2}-\frac{1}{2 \sqrt{1-4 \lambda}}\right)(t+1)^{\frac{1}{2} \sqrt{1-4 \lambda}}+\left(\frac{1}{2}-\frac{1}{2 \sqrt{1-4 \lambda}}\right)(t+1)^{-1 / 2 \sqrt{1-4 \lambda}}\right]$

for $\lambda<\frac{1}{4}$ and

$$
x(t)=(t+1)^{-1 / 2}\left[\cos \left(\frac{1}{2} \sqrt{4 \lambda-1} \ln (t+1)\right)-\frac{1}{\sqrt{4} \lambda-1} \sin \left(\frac{1}{2} \sqrt{4 \lambda-1}\right) \ln (t+1)\right]
$$

for $\lambda>\frac{1}{4}$. Hence the boundary value problem

$$
\left((t+1)^{2} x^{\prime}(t)\right)^{\prime}+\lambda x(t)=0, \quad x^{\prime}(0)=0, x(\infty)=0
$$

has no solution for any $\lambda \leq 0$, while every $\lambda>0$ is an eigenvalue with the corresponding eigenfunction $x(t)$.

Remark 1.4. The foregoing examples indicate that if condition (1.2) is violated, then we have either no eigenvalue at all (Example 1.2), or we have a continuum of eigenvalues, and the spectrum is bounded from below but not discrete (Example 1.3). This seems to support the conjecture formulated in Drábek and Kufner [4, 
Section 3], where the discreteness of the spectrum is connected with the behaviour of the product of integrals in (1.2). See also Remark 2.2 below.

\section{The functional analytic Setting}

Let $L^{p}(c)$ denote the weighted Lebesgue space of all functions $x=x(t)$ defined on $(0, \infty)$, for which

$$
\|x\|_{p ; c}:=\left(\int_{0}^{\infty} c(t)|x(t)|^{p} \mathrm{~d} t\right)^{1 / p}
$$

is finite. Then $L^{p}(c)$ equipped with the norm $\|\cdot\|_{p ; c}$ is a uniformly convex Banach space.

Let $W_{\infty}^{1, p}(r)$ be the set of all absolutely continuous functions $x=x(t)$ defined on $[0, \infty)$ such that $x(\infty)=0$ and

$$
\|x\|_{1, p ; r}:=\left(\int_{0}^{\infty} r(t)\left|x^{\prime}(t)\right|^{p} \mathrm{~d} t\right)^{1 / p}
$$

is finite. Then $W_{\infty}^{1, p}(r)$ equipped with the norm $\|\cdot\|_{1, p ; r}$ is a uniformly convex Banach space.

A function $x \in W_{\infty}^{1, p}(r)$ is called a weak solution of (1.1) if the integral identity

$$
\int_{0}^{\infty} r(t) \varphi\left(x^{\prime}(t)\right) y^{\prime}(t) \mathrm{d} t=\lambda \int_{0}^{\infty} c(t) \varphi(x(t)) y(t) \mathrm{d} t
$$

holds for all $y \in W_{\infty}^{1, p}(r)$ (with both integrals being finite). Let us note that if $x \in W_{\infty}^{1, p}(r)$ is a weak solution of (1.1), then it is also a solution of (1.1) in the sense mentioned in Section 1. Indeed, assume that $x \in W_{\infty}^{1, p}(r)$ is a weak solution; then, in particular, (2.1) holds for all $y \in C_{0}^{\infty}[0, \infty)$. Integration by parts yields

$$
\int_{0}^{\infty}\left[r(t) \varphi\left(x^{\prime}(t)\right)-\int_{0}^{t} \lambda c(\tau) \varphi(x(\tau)) \mathrm{d} \tau\right] y^{\prime}(t) \mathrm{d} t=0,
$$

i.e., the distributional derivative of the expression in brackets is equal to zero. Hence there exists a real constant $C$ such that

$$
r(t) \varphi\left(x^{\prime}(t)\right)-\int_{0}^{t} \lambda c(\tau) \varphi(x(\tau)) \mathrm{d} \tau=C
$$

a.e. in $(0, \infty)$. However, continuity of $c(\tau) \varphi(x(\tau))$ implies that $(2.2)$ holds for all $t \in[0, \infty)$, and $r \varphi\left(x^{\prime}\right) \in C^{1}[0, \infty)$.

The reason for considering weak solutions consists of reformulating (1.1) as an abstract nonlinear eigenvalue problem and finding the eigenvalues employing the tools of nonlinear functional analysis. Oscillation theory and other properties of the initial value problem associated with the equation in (1.1) then show that these eigenvalues and the corresponding eigenfunctions have the properties stated in Theorem 1.1.

In order to use compactness arguments associated with the Palais-Smale condition (see Section 4 for more details) we need the compact embedding:

$$
W_{\infty}^{1, p}(r) \hookrightarrow \hookrightarrow L^{p}(c) .
$$

This compactness result holds if and only if condition (1.2) is satisfied. Indeed, it follows from the results of Opic and Kufner [8, Theorem 7.13 and Remark 7.14] 
that (2.3) holds if and only if (1.2) and also

$$
\lim _{t \rightarrow 0}\left(\int_{0}^{t} c(\tau) \mathrm{d} \tau\right)^{1 / p}\left(\int_{t}^{\infty} r^{1-p^{\prime}}(\tau) \mathrm{d} \tau\right)^{1 / p^{\prime}}=0
$$

are satisfied. However, the weight functions $r$ and $c$ are much more general in 8 than in our situation. Namely, the continuity of $r$ and $c$ in $[0, \infty)$ implies that (2.4) is always satisfied in our case.

Having the compact embedding (2.3) available, we can apply the Lagrange multiplier method to get the following assertion.

Lemma 2.1. Let us assume (1.2). Then (1.1) has the least eigenvalue $\lambda_{1}>0$ which can be characterized as follows:

$$
\lambda_{1}=\min \frac{\int_{0}^{\infty} r(t)\left|x^{\prime}(t)\right|^{p} \mathrm{~d} t}{\int_{0}^{\infty} c(t)|x(t)|^{p} \mathrm{~d} t}
$$

where the miminum is taken over all $x \in W_{\infty}^{1, p}(r), x \neq 0$.

Remark 2.2. It is interesting to point out that if (1.2) is violated but

$$
\sup _{t \in(0, \infty)}\left(\int_{0}^{t} c(\tau) \mathrm{d} \tau\right)^{1 / p}\left(\int_{t}^{\infty} r^{1-p^{\prime}}(\tau) \mathrm{d} \tau\right)^{1 / p^{\prime}}<\infty
$$

holds, then a continuous embedding $W_{\infty}^{1, p}(r) \hookrightarrow L^{p}(c)$ takes place instead of a compact one (see, e.g., Opic and Kufner [8, Theorem 1.14]). This property, however, is not sufficient to make our approach work.

On the other hand, condition (2.6) guarantees the boundedness of the spectrum from below (see, e.g., Drábek and Kufner [4. Section 1]), and this condition is satisfied in Example 1.3 and violated in Example 1.2. Let us point out that condition (2.6) was discovered by G. Talenti, G. Tomaselli, B. Muckenhoupt and others as a necessary and sufficient condition for a weighted Hardy inequality which is equivalent to the embedding (2.3). For details, see [8].

\section{The initial value problem}

Let $t_{0} \in[0, \infty), A, B \in \mathbb{R}$. Consider the initial value problem

$$
\left\{\begin{array}{l}
\left(r(t) \varphi\left(x^{\prime}(t)\right)\right)^{\prime}+\lambda c(t) \varphi(x(t))=0, \quad t>t_{0}, \\
x\left(t_{0}\right)=A, \quad x^{\prime}\left(t_{0}\right)=B
\end{array}\right.
$$

Using the generalized Prüfer transformation and making use of the generalized Elbert's sine function (see Elbert [7, p. 155]), one can prove that there exists a unique solution of $(3.1)$ which is extensible over the whole interval $[0, \infty)$ (the notion of the solution is defined here as in Section 1). In this section we will emphasize some properties of this solution which will be used in the proof of our main result in Section 4.

Proposition 3.1 (see Došlý [3, Theorem 2.12]). The following statements are equivalent:

(i) The equation in (3.1) is disconjugate on an interval $[a, b] \subset[0, \infty)$, i.e. any nontrivial solution of the equation in (3.1) has at most one zero in $[a, b]$.

(ii) There exists a solution of the equation in (3.1) having no zero in $[a, b]$. 
(iii) The functional

$$
\mathcal{F}(y ; a, b):=\int_{a}^{b}\left[r(t)\left|y^{\prime}(t)\right|^{p}-\lambda c(t)|y(t)|^{p}\right] \mathrm{d} t
$$

is positive for every $y \in W_{0}^{1, p}(a, b), y \neq 0$ (here $W_{0}^{1, p}(a, b)$ denotes the usual Sobolev space of functions $y$ on $(a, b)$ with $y(a)=y(b)=0)$.

Remark 3.2. The equivalence (i) $\Longleftrightarrow$ (ii) is in fact the Sturm separation theorem for (3.1) while the equivalence (i) $\Longleftrightarrow$ (iii) is the Sturm comparison theorem for (3.1). In particular, we have the following two assertions.

Corollary 3.3. Let $x_{i}=x_{i}(t), t \in[0, \infty), i=1,2$, be two solutions of (3.1) with $t_{0}=0, A=1, B=0$ and $\lambda=\mu_{i} ; i=1,2,0<\mu_{1}<\mu_{2}$. Let $t_{i} \in(0, \infty)$ be the first zero of $x_{i}, i=1,2$. Then $t_{2}<t_{1}$.

Corollary 3.4. Let $x_{i}=x_{i}(t), i=1,2$, be as in Corollary 3.3. Then between two consecutive zeros of $x_{1}$ there is at least one zero of $x_{2}$.

The above assertions allow us to compare zeros of solutions in finite intervals "near zero". In order to compare zeros "near infinity", we need the notion of nonoscillatory solutions. Namely, the equation in (3.1) is called nonoscillatory if for any nontrivial solution $x=x(t)$ of this equation there exists $T=T(x)>0$ such that $x(t) \neq 0$ for all $t \in[T, \infty)$. If this is not true, the equation in (3.1) is called oscillatory.

Proposition 3.5 (see Došlý [2] or [3, Theorem 2.13]). Let

$$
\limsup _{t \rightarrow \infty}\left(\int_{t}^{\infty} r^{1-p^{\prime}}(\tau) \mathrm{d} \tau\right)^{p-1}\left(\int_{0}^{t} c(\tau) \mathrm{d} \tau\right)<\frac{(p-1)^{p-1}}{p^{p}} .
$$

Then the equation in (3.1) with $\lambda=1$ is nonoscillatory. Let

$$
\liminf _{t \rightarrow \infty}\left(\int_{t}^{\infty} r^{1-p^{\prime}}(\tau) \mathrm{d} \tau\right)^{p-1}\left(\int_{0}^{t} c(\tau) \mathrm{d} \tau\right)>\frac{(p-1)^{p-1}}{p^{p}} .
$$

Then the equation in (3.1) with $\lambda=1$ is oscillatory.

Corollary 3.6. Let us assume (1.2). Then the equation in (3.1) is nonoscillatory for any $\lambda \in \mathbb{R}$. Let us assume that (1.2) does not hold, i.e., there is a sequence $t_{n} \rightarrow \infty($ as $n \rightarrow \infty)$ such that

$$
\liminf _{t_{n} \rightarrow \infty}\left(\int_{0}^{t_{n}} c(\tau) \mathrm{d} \tau\right)^{1 / p}\left(\int_{t_{n}}^{\infty} r^{1-p^{\prime}}(\tau) \mathrm{d} \tau\right)^{1 / p^{\prime}}>0 .
$$

Then there exists $\lambda_{0}>0$ such that the equation in (3.1) is oscillatory provided $\lambda \geq \lambda_{0}$.

Lemma 3.7. Let us assume that (1.2) holds and let $x_{i}=x_{i}(t), t \in[0, \infty), i=1,2$, be as in Corollary 3.3. Then $x_{i}$ has the largest zero $\xi_{i} \in(0, \infty), i=1,2$, and $\xi_{2}>\xi_{1}>0$.

Proof. The existence of $\xi_{i}, i=1,2$, follows from Corollary 3.6; the inequality $\xi_{2}>\xi_{1}$ then follows from Proposition 3.1(iii).

Corollary 3.8. Let us assume that (1.2) holds and let $x=x(t)$ be a nontrivial solution of (3.1). Then $x$ has at most a finite number of zeros in $(0, \infty)$. 
Proof. There is the largest zero $\xi$ of $x$ by Corollary 3.6. The assertion now follows from the compactness of the interval $[0, \xi]$ and from the uniqueness of the solution of the initial value problem (3.1).

\section{The eigenvalue problem}

The following two assertions are consequences of the properties of the initial value problem (3.1). The first follows from the uniqueness of the solution and the homogeneity of the equation in (3.1).

Lemma 4.1. Let us assume (1.2). Then every eigenvalue of (1.1) is simple, i.e., for any eigenvalue $\lambda$ of (1.1) all associated eigenfunctions $x_{\lambda}$ are mutually proportional.

The second assertion follows directly from Corollaries 3.3, 3.4, 3.8 and from Lemma 3.7.

Lemma 4.2. Let us assume (1.2) and let $\bar{\lambda}, \hat{\lambda}$ be two eigenvalues of (1.1) such that $\bar{\lambda}>\widehat{\lambda}$ and $x_{\bar{\lambda}}, x_{\widehat{\lambda}}$ are associated eigenfunctions, respectively. Then the number of zeros of $x_{\bar{\lambda}}$ in $(0, \infty)$ is strictly larger than the number of zeros of $x_{\hat{\lambda}}$ in $(0, \infty)$.

Proposition 4.3. Let us assume (1.2). Then the set of all eigenvalues of (1.1) consists of isolated points in $(0, \infty)$.

Proof. Let $\lambda_{0}$ be a limit point of a sequence $\left\{\lambda_{k}\right\}$ of eigenvalues. We can extract either a monotone increasing or a monotone decreasing (or both) subsequence converging to $\lambda_{0}$. Denote it again by $\left\{\lambda_{k}\right\}$. If $\left\{\lambda_{k}\right\}$ is monotone increasing and $\lambda_{k} \rightarrow \lambda_{0}$, then the number of zeros of $x_{\lambda_{k}}$ increases with $k$ (Lemma 4.2) so $x_{\lambda_{0}}$ has more zeros than any $x_{\lambda_{k}}$ contradicting Corollary 3.8. In the case that $\left\{\lambda_{k}\right\}$ is a decreasing sequence, by Corollary $3.8 x_{\lambda_{0}}$ has finitely many zeros and by Lemma 4.2 the number of zeros of $x_{\lambda_{k}}$ is a strictly decreasing sequence as $k \rightarrow \infty$, which is impossible. So the sequence must be increasing and have the limit $\infty$.

Let $\mathcal{S}:=\left\{x \in W_{\infty}^{1, p}(r):\|x\|_{p ; c}=1\right\}$. For a closed and symmetric set $\mathcal{A} \subset \mathcal{S}$ define the Krasnoselskij genus

$\gamma(\mathcal{A}):=\inf \left\{m \in \mathbb{N}: \exists\right.$ continuous and odd mapping of $\mathcal{A}$ into $\left.\mathbb{R}^{m} \backslash\{0\}\right\}$,

$\gamma(\mathcal{A}):=\infty$ if no such $m$ exists.

Define a family of sets

$$
\mathcal{F}_{k}^{*}:=\{\mathcal{A} \subset \mathcal{S}: \mathcal{A}=-\mathcal{A}, \quad \gamma(\mathcal{A})=k\}, \quad k \in \mathbb{N} .
$$

Let $\mathcal{S}^{k}$ be the unit sphere in $\mathbb{R}^{k}$. For $k \in \mathbb{N}$ define a family of sets $\mathcal{F}_{k}:=\{\mathcal{A} \subset \mathcal{S}$ :

$\mathcal{A}=-\mathcal{A}, \mathcal{A}=h\left(\mathcal{S}^{k}\right)$ with $h$ a continuous odd mapping from $\mathbb{R}^{k}$ into $\left.\mathcal{S}\right\}$.

It follows easily that

$$
\mathcal{F}_{k} \subset \mathcal{F}_{k}^{*} \text {. }
$$

Let us consider the functional

$$
I(x):=\|x\|_{1, p ; r}^{p} .
$$

The compact embedding (2.3) allows to prove that for any $k \in \mathbb{N}$,

$$
\begin{aligned}
\lambda_{k}^{*} & =\min _{\mathcal{A} \in \mathcal{F}_{k}^{*}} \max _{x \in \mathcal{A}} I(x), \\
\lambda_{k} & =\min _{\mathcal{A} \in \mathcal{F}_{k}} \max _{x \in \mathcal{A}} I(x)
\end{aligned}
$$


are eigenvalues of (1.1). The interested reader is referred to Drábek and Robinson [5, Section 3] for the proof which can be literally adapted in our situation.

It follows from (4.1) that $\lambda_{k}^{*} \leq \lambda_{k}, k=1,2, \ldots$ Exactly as in [5, p. 195] we can also prove that $\lambda_{i}^{*}=\lambda_{i}$ for $i=1,2, \lambda_{1}<\lambda_{2}$, and that $x_{\lambda_{1}}$ has no zero and $x_{\lambda_{2}}$ has exactly one zero in $(0, \infty)$. It is known (cf. Cuesta [1, Section 4.3]) that the Ljusternik-Schnirelman eigenvalues $\lambda_{k}^{*}$ satisfy $\lambda_{k}^{*} \rightarrow \infty$ as $k \rightarrow \infty$. Hence also $\lambda_{k} \rightarrow \infty$. This fact, nonetheless, follows also from Proposition 4.3.

Note also that every set $\mathcal{A} \in \mathcal{F}_{1}$ is formed by two antipodal points from $\mathcal{S}$ and so for $k=1$ both characterizations (4.2) and (4.3) coincide with (2.5).

Proposition 4.4. Let (1.2) be satisfied. The sequences defined by (4.2) and (4.3) coincide, i.e. $\lambda_{k}^{*}=\lambda_{k}$ for all $k=1,2, \ldots$, and the sequence $\left\{\lambda_{k}\right\}_{k=1}^{\infty}$ is the entire set of all eigenvalues of (1.1). The eigenfunction $x_{\lambda_{n}}$ associated with $\lambda_{n}$ has precisely $n-1$ zeros in $(0, \infty)$, and between two consecutive zeros of $x_{\lambda_{n-1}}$ there is exactly one zero of $x_{\lambda_{n}}$.

Proof. Let us show first that all eigenvalues $\lambda_{k}^{*}, k=1,2, \ldots$, are mutually different. As mentioned above, $\lambda_{1}^{*}<\lambda_{2}^{*}$ (cf. [5, Section 3]). Assume by contradition that for some $k \geq 2, j \geq 1$, we have

$$
\lambda_{k}^{*}=\lambda_{k+1}^{*}=\cdots=\lambda_{k+j}^{*} .
$$

The result of Szulkin [9] or Cuesta [1, Theorem 4.2] implies that for the set $\mathcal{A}_{j} \subset \mathcal{S}$ of all eigenfunctions associated with $\lambda_{k}^{*}$ we have $\gamma(\mathcal{A} j) \geq j+1$. In particular, this means that the set of all eigenfunctions associated with $\lambda_{k}^{*}$ and normalized by the condition $\left\|x_{\lambda_{k}^{*}}\right\|_{p ; c}=1$ consists of more than two antipodal points. However, this contradicts Lemma 4.1 .

Secondly, we prove that $\lambda_{k}^{*}=\lambda_{k}$ for all $k=1,2, \ldots$ Following literally the result of Drábek and Robinson [6, Section 3] one can prove that the eigenfunction $x_{\lambda_{n}}$ associated with the eigenvalue $\lambda_{n}$ has at most $n-1$ zeros in $(0, \infty)$. As a consequence of this fact and Lemmas 4.1, 4.2 we obtain that $\lambda_{k}^{*}=\lambda_{k}$ for all $k \in \mathbb{N}$ and that $x_{\lambda_{n}}$ has precisely $n-1$ zeros in $(0, \infty)$. At the same time, Lemma 4.2 implies that there are no other eigenvalues than those defined by (4.2) or (4.3).

The interlacing property of zeros of $x_{\lambda_{n-1}}$ and $x_{\lambda_{n}}$ then follows from Corollary 3.3 and Lemma 3.7.

Proof of Theorem 1.1. This is immediate from Propositions 4.3, 4.4 and Lemma 4.1.

The authors are grateful to the referee for his comments which substantially improved this paper.

\section{REFERENCES}

[1] M.Cuesta: On the Fučík Spectrum of the Laplacian and the p-Laplacian. In: Proceedings of Seminar in Differential Equations (P. Drábek, ed.), Univ. of West Bohemia Pilsen (2000), 67-96.

[2] O. Došlý: Oscillation criteria for half-linear second order differential equations, Hiroshima J. Math. 28 (1998), 507-521. MR1657543 (99j:34044)

[3] O. Došlý: Oscillation Theory of Linear Differential and Difference Equations. In: Proceedings of Seminar in Differential Equations (P. Drábek, ed.), Univ. of West Bohemia Pilsen (2002), 7-71.

[4] P. Drábek and A. Kufner: Note on spectra of quasilinear equations and the Hardy inequality. In: Nonlinear Analysis and Applications (R. P. Agarwal and D. O'Regan, eds.), Vol. I. Kluwer Academic Publishers, Dordrecht 2003, 505-512. MR2060230 
[5] P. Drábek and S. Robinson: Resonance problems for the p-Laplacian, J. Functional Analysis 169 (1999), 189-200. MR.1726752 (2000j:35096)

[6] P. Drábek and S. Robinson: On the generalizations of the Courant nodal domain theorem, J. Differential Equations 181 (2002), 58-71. MR1900460 (2003g:35170)

[7] Á. Elbert: A half-linear second order differential equation, Colloq. Math. Soc. János Bolyai 30 (1979), 153-180. MR0680591 (84g:34008)

[8] B. Opic and A. Kufner: Hardy-Type Inequalities, Pitman Research Notes in Mathematics Series 279, Longman Scientific \& Technical, Harlow 1990. MR1069756 (92b:26028)

[9] A. Szulkin: Ljusternik-Schnirelman theory on $C^{1}$ manifolds, Ann. Inst. Henri Poincaré, Vol. 5, No.2 (1988), 119-139. MR0954468 (90a:58027)

Department of Mathematics, University of West Bohemia, Univerzitní 22, 30614 Plzeñ, Czech Republio

E-mail address: pdrabek@kma.zcu.cz

Department of Mathematics, University of West Bohemia, Univerzitní 22, 30614 Plzeñ, Czech Republic

E-mail address: kufner@math.cas.cz 